\title{
HD 4915: A Maunder Minimum Candidate
}

\author{
Shivani P. Shah ${ }^{1}$ (1), Jason T. Wright ${ }^{1}$ (1) , Howard Isaacson $^{2}$ (1) , Andrew W. Howard ${ }^{3}$ (1) , and Jason L. Curtis $^{4,5}$ (1) \\ ${ }^{1}$ Center for Exoplanets and Habitable Worlds, Department of Astronomy \& Astrophysics, \\ The Pennsylvania State University, 525 Davey Laboratory, University Park, PA 16802, USA \\ ${ }^{2}$ Department of Astronomy, 601 Campbell Hall, University of California, Berkeley, CA 94720-3411, USA \\ ${ }^{3}$ California Institute of Technology, 1200 East California Boulevard, Pasadena, CA, 91125, USA \\ ${ }^{4}$ Department of Astronomy, Columbia University, 550 West 120th Street, New York, NY 10027, USA \\ Received 2018 January 29; revised 2018 June 13; accepted 2018 June 28; published 2018 August 16
}

\begin{abstract}
We study the magnetic activity cycle of HD 4915 using the Ca II HK emission line strengths measured by Keck I/ High Resolution Echelle Spectrometer (HIRES) spectrograph. The star has been observed as a part of California Planet Search Program from 2006 to present. We note decreasing amplitude in the magnetic activity cycle, a pattern suggesting the star's entry into a Magnetic Grand Minimum state, reminiscent of the Sun's Maunder and Dalton Minima. We recommend further monitoring of the star to confirm the grand minimum nature of the dynamo, which would provide insight into the state of the Sun's chromosphere and the global magnetic field during its grand minima. We also recommend continued observations of HK emission lines, and ground- or spacebased photometric observations to estimate the sunspot coverage.
\end{abstract}

Key words: magnetic fields - stars: activity - stars: chromospheres - Sun: activity - sunspots

Supporting material: data behind figures

\section{Introduction}

It has been almost 175 years since Heinrich Schwabe discovered the 11-year sunspot cycle in 1843. With the development of solar dynamo theory as the mechanism responsible for creating the Sun's magnetic field, the sunspot cycle was realized to be a manifestation of the Sun's magnetic cycle (see Charbonneau 2014 for a review). About 140 years after the discovery of sunspot cycle, Eddy (1976) compiled strong historical evidence spanning centuries to show that the "prolonged sunspot minimum" period from 1645 to 1715 , as speculated by Gustav Spörer in 1889 and E. W Maunder in 1894, was real. Eddy called this period the Maunder Minimum (MM). Two other such periods, although less dramatic, were identified by the community (e.g., Siscoe 1980), the Dalton (1796-1820) and Spörer Minima (1460-1550). ${ }^{6}$ Such periods are now more generally termed Magnetic Grand Minima (MGM; e.g., Saar \& Testa 2012) and are characterized by repressed magnetic activity that lasts longer than at least the typical cycle of the star.

Learning more about MGM periods has been of interest because it addresses one of the many elusive characteristics of the solar dynamo, thereby contributing to our understanding of the Sun's magnetic field, especially its evolution in the past and future. To maximize this learning, several surveys of Sun-like stars have been conducted, with the goal of collecting a large sample of stars in MGM states.

Today, the standard metric for sunspot coverage is group sunspot number (GSN), which uses the number of groups of sunspots to provide a more self-consistent and less noisy data set than the previous calibrations (Hoyt \& Schatten 1998). Because it is challenging to observe sunspots on Sun-like stars on decade timescales, it is necessary to use a proxy for global magnetic field strength instead, such as chromospheric line

\footnotetext{
5 NSF Astronomy \& Astrophysics Postdoctoral Fellow.

6 The origin of the term "Dalton Minimum" is unclear, but the first usage we are aware of is in Eddy (1982). Spörer Minimum was first used in Eddy (1976).
}

emission. In this regard, the most common proxy has been the emission cores of the $\mathrm{Ca}$ II $\mathrm{H}$ and $\mathrm{K}$ lines at $3968 \AA$ and $3934 \AA$, respectively.

A drawback of using this proxy is the absence of chromospheric line emission data from the MGM periods of the Sun, specifically the MM, preventing an absolute comparison in the activity of the Sun and other stars. This compels the use of sunspots instead; however, it is important to note that although the sunspots have shown an almost flat activity during the MM period, other solar proxies, including the cosmogenic isotopes (Kocharov 1986), have in fact indicated a weakly cycling activity.

\subsection{Searching for MGM in Other Stars}

The oldest survey to track the Ca II HK line emission was the Mount Wilson Observatory HK Project that examined bright nearby stars for photospheric and chromospheric variability for decades (Wilson 1968; Vaughan et al. 1978; Baliunas et al. 1995). With the extensive data available through this survey, the search for MGM candidates followed; this search has now spanned decades, using data from some of the most extensive surveys. The biggest challenge, however, has been to set down the criteria for a good MGM candidate based on observations of magnetic activity levels, metallicity, age, stellar type, gravity, and the relation between these properties (e.g., Baliunas \& Jastrow 1990; Henry et al. 1996; Saar \& Testa 2012).

One of the earliest attempts to set down the criteria was made by Baliunas \& Jastrow (1990), as they investigated the magnetic activity distribution of 74 solar-type stars from the Mount Wilson HK Project using the Mount Wilson S-Index measurement. Their selection criteria for Sun-like stars were: (1) $B-V$ in the range of 0.60-0.76 (the Sun has $B-V=0.65$ ), and (2) an age similar to the Sun.

Baliunas \& Jastrow (1990) found that the frequency distribution of these 74 stars had a distinct bimodal feature with a broad peak centered at $S \approx 0.17$ and a narrow peak centered at $S \approx 0.145$, lower than the S-Index of Sun's typical 
minimum, $S=0.164$ (Figure 2 in Baliunas \& Jastrow 1990). They suggested that the broad peak represented stars undergoing cyclic variations like the Sun and the narrow peak represented stars in Maunder minimum, a term used to describe stars in a state like the MM of the Sun. They noted that four of these Maunder minimum candidates showed "flat" activity time series, concluding that $30 \%$ of Sun-like stars are in states analogous to MM of the Sun.

Based on this, Baliunas \& Jastrow (1990) proposed that future Maunder minimum candidates could be identified by a combination of "flat" activity and an average $S<0.15$; i.e., they should contribute to the narrow peak.

Henry et al. (1996) conducted a similar investigation, but on a sample of stars surveyed as a part of the Project Phoenix Survey (Cullers 2000), and found a similar bimodal distribution. Henry et al. (1996) further formed a subclass of very inactive stars with $\log R_{\mathrm{HK}}^{\prime}<-5.1$, corresponding to $S \approx 0.15$.

However, using accurate parallaxes from the Hipparcos Catalog (Perryman et al. 1997), Wright (2004) showed that almost all Maunder minimum candidates in the Mount Wilson and Project Phoenix surveys were actually of luminosity class IV/V or IV (i.e., either slightly evolved or subgiants). As had been shown by do Nascimento et al. (2003), evolved stars generally have lower S-Indices than dwarf stars of the same color, and at any rate evolved stars are, by definition, not Sun-like, and so do not belong in these samples. Because the effects of modest evolution are only weakly discernable in stellar spectra (apparent only as subtle changes in gravitysensitive lines), surveys of Sun-like stars conducted prior to Hipparcos suffered significant contamination from subgiants due to the Malmquist bias.

As a part of another effort, Giampapa et al. (2006) found in their survey of the M67 cluster that $17 \%$ of Sun-like stars exhibited noticeably smaller magnetic activity levels than the typical levels of the Sun, and categorized them to be Maunder minimum candidates. Giampapa et al. (2006) addressed this phenomenon qualitatively and suggested that Maunder minimum-like periods were low-amplitude extensions of the solar dynamo rather than a state in a separate modal frequency, as was indicated by Baliunas \& Jastrow (1990) and Henry et al. (1996).

However, Curtis (2017) demonstrated that the low activity levels were an effect of the contamination of the HK lines due to calcium absorption in the ISM. On correcting the HK line strengths for likely levels of ISM absorption, Curtis (2017) concluded in his analysis that none of the stars were MM candidates.

Saar \& Testa (2012) also attempted to set down the criteria for classifying an MGM candidate; this analysis was inspired by Wright (2004)'s question of whether $R_{\mathrm{HK}}^{\prime}$ was calibrated correctly, as it did not account for the gravity and metallicity of the star. Saar \& Testa (2012) investigated the relationship between $R_{\mathrm{HK}}^{\prime}$ and metallicity, and they found that indeed the most inactive metal-rich stars had lower $R_{\mathrm{HK}}^{\prime}$ values than the most inactive metal-poor stars. Saar \& Testa (2012) set down new criteria for MGM candidates: (1) the star is a bona fide dwarf, (2) the $R_{\mathrm{HK}}^{\prime}$ of the star should be lower than a threshold set 0.054 dex above the minimum seen for stars of similar metallicity, and (3) the $\mathrm{HK}$ variation as given by $\sigma / S_{\mathrm{HK}} \leqslant 2 \%$ for a time period spanning $\geqslant 4$ years of measurements. Thus,

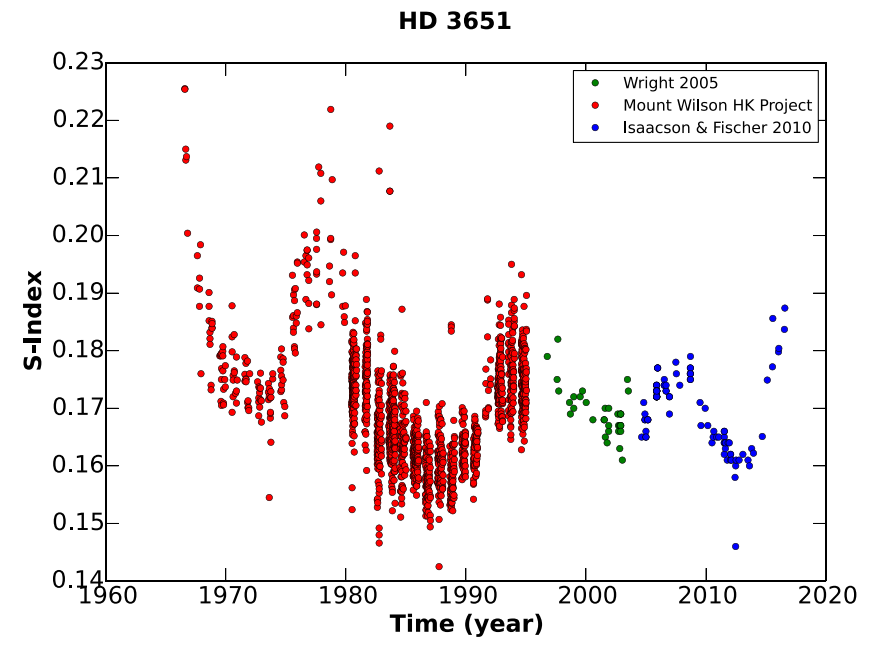

Figure 1. S-Index time series of HD 3651 from the Mount Wilson HK Project (1965-1995, red), Wright (2004; 1996-2004, green), and Isaacson \& Fischer (2010; 2004-present, blue) to show persistent magnetic activity cycles. Based on the red points, Donahue et al. (1995) suggested that the star might be entering a Maunder minimum-like state, but subsequent monitoring at Keck Observatory shows that the star continues to cycle. Note that in general there may be differences in the calibration of the three data sets, although based on this figure there do not seem to be any obvious discrepancies for this star. The data used to create this figure are available.

they implicitly assumed that MGMs are rare and characterized by extraordinary $R_{\mathrm{HK}}^{\prime}$ values.

The use of chromospheric activity time series as a discriminant of an MGM, as opposed to using an absolute chromospheric activity activity level, is advantageous in that it would allow us to determine the appropriate threshold level when some such stars are found if, indeed, such a level even exists. It also allows us to tie the search criteria for a MGM to the historical sunspot records, instead of proxies for the overall activity level during the Sun's MGM. The success of this method then depends on $R_{\mathrm{HK}}^{\prime}$ being a good proxy for sunspot number during the MGM.

\section{2. $H D 3651$}

One such attempt to identify a MM analog via chromospheric time series is that of Donahue et al. (1995), who identified HD 3651 as star that might be "entering a Maunder minimum phase" using the S-Index values from the Mount Wilson Observatory HK Project (see Figure 1). Subsequent monitoring at Keck Observatory as part of the California Planet Survey shows that the star has continued to cycle, although apparently at a much lower amplitude and about a mean significantly higher than the 1987 magnetic minimum.

So in this case, Donahue et al. (1995)'s prediction that a star that appeared to have a strongly decaying activity cycle was entering a Maunder minimum state does not seem to have been validated, although the star does seem to be in a more weakly cycling state.

\section{Data}

In order to compare results with the seminal Mt. Wilson survey, HK emission is usually expressed in the instrumental unit used by that survey, the S-Index, which essentially tracks the equivalent width of the emission in the HK line cores (Vaughan et al. 1978). The strength of these Ca II emission lines is proportional to the heating of the Sun's chromosphere 

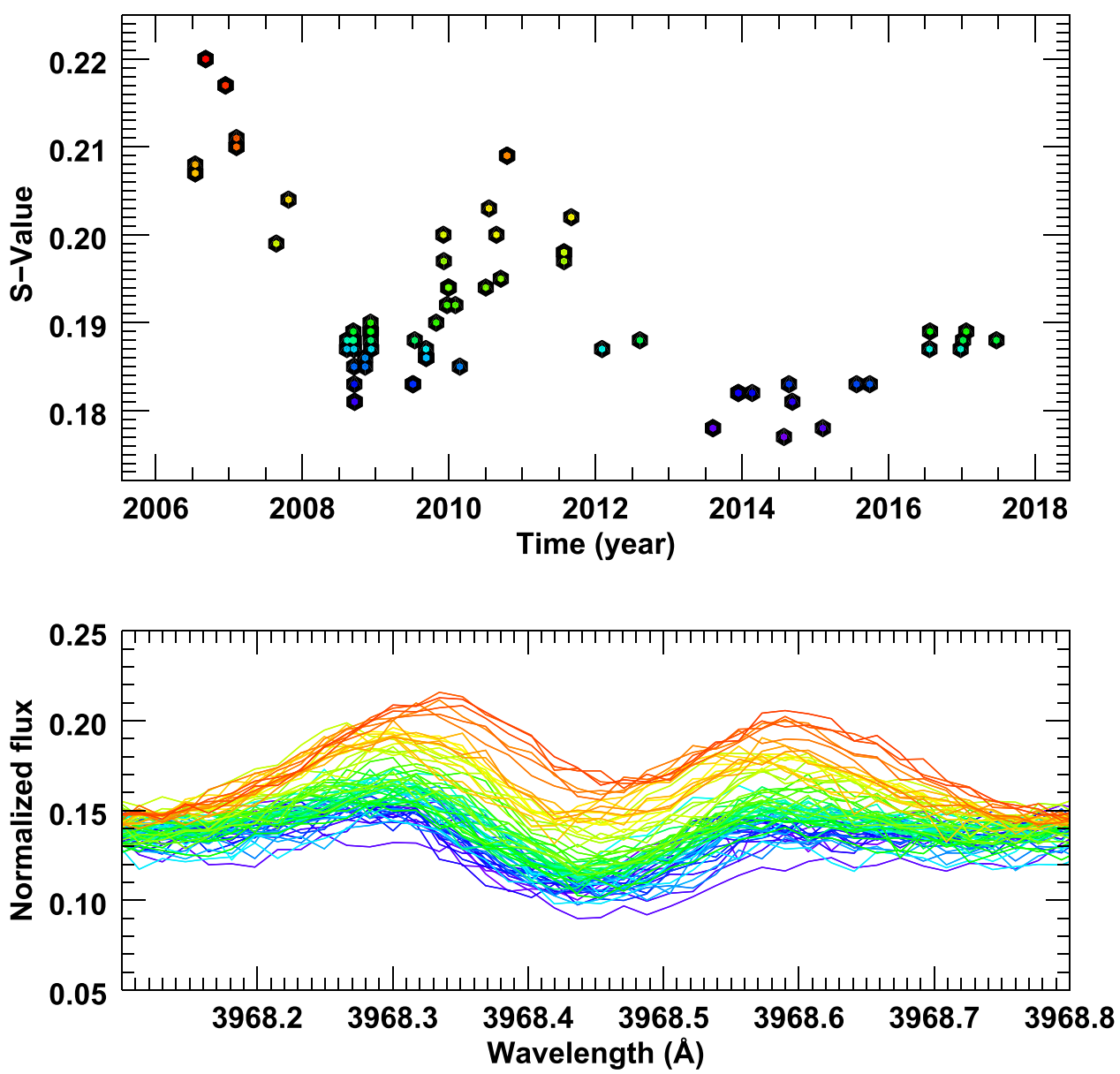

Figure 2. S-Indices of HD 4915 from 2006 until 2017 (top panel). The symbols are color coded according to their value. The $Y$-axis represents the S-Indices as calculated by Isaacson \& Fischer (2010) with an uncertainty of \pm 0.002 , and the $X$-axis represents time in years. We see a trend of decreasing maxima as the magnetic activity of the star completes two cycles. We also note that the second minimum is lower than the first. The colors of the data points correspond to the respective colored spectrum in the spectral diagram of the star (bottom panel). The $Y$-axis of the spectral diagram represents normalized flux. and the $X$-axis represents wavelength. The spectra is zoomed in to show Ca II H emission line at $\sim 3968.4 \AA$ (an indicator of magnetic activity). The color coordination of the S-Indices with the spectra confirm the trend of decreasing amplitude of magnetic activity cycle. The data used to create this figure are available.

by the magnetic fields on the surface and has a direct correspondence with the strength of the magnetic activity and the area covered (Leighton 1959; Skumanich et al. 1975). Another commonly used index is $R_{\mathrm{HK}}^{\prime}$, which is the ratio of the chromospheric emission in $\mathrm{HK}$ lines to the total bolometric emission from the star so that it is independent of temperature and photospheric component of the star (Noyes et al. 1984).

For our analysis, we have used the HD 4915 spectra from 2006 until present, collected as a part of the California Planet Search (CPS) Radial Velocity Survey (Howard et al. 2010) using Keck/High Resolution Echelle Spectrometer (HIRES; Vogt et al. 1994). The spectra are publicly available on Keck Observatory Archive. ${ }^{7}$ For data prior to 2010 we have taken data directly from the tables of Isaacson \& Fischer (2010) and the calculated the rest (post-2010) from CPS spectra using the same methodology used to generate those tables.

Because the Butler et al. (2017) analysis of these same spectra find a slightly different S-Index time series that does not as obviously show evidence of entry into an MGM state, we have examined the spectra themselves to confirm that our S-Index measurements accurately reflect the emission in the

\footnotetext{
7 https://koa.ipac.caltech.edu
}

HK lines. The top panel of Figure 2 shows the S-Index time series color coded by value, and the bottom panel shows the corresponding $\mathrm{Ca}$ II $\mathrm{H}$ line core spectra. We show this colorcoded correspondence to confirm a faithful tracking of the total emission in the line core by our S-Indices.

\section{HD 4915}

HD 4915 is a G5V star with an effective temperature of $5668 \mathrm{~K}$, $\log g=4.57$, vsini of $1.4 \mathrm{~km} \mathrm{~s}^{-1}$, [M/H] of $-0.2 \mathrm{dex}$, and an isochrone derived age in the range of 0.6-4.4 Gyrs (Brewer et al. 2016). These spectroscopic properties of HD 4915 differ from that of the Sun's by -104 K, 0.132 dex (Prša et al. 2016), and $0.2 \mathrm{~km} \mathrm{~s}^{-1}$ (Pavlenko et al. 2012), respectively, classifying it as a dwarf and also Sun-like.

Over its last cycle, which we suspect to be its transition period into an MGM state, it has an average S-Index of 0.184 and and HK variation of $2.28 \%$. Following the analysis done by Noyes et al. (1984) for transforming S-Index to $R_{\mathrm{HK}}^{\prime}$, and using $B-V=0.66$ (Soubiran et al. 2016), we compute $\log R_{\mathrm{HK}}^{\prime}=-4.872$.

We might use the star's activity level to deduce an estimate of its age using the activity-rotation-age relation formulated by Mamajek \& Hillenbrand (2008), but the star's apparent entry into an MGM state confuses this, because that relation is based 

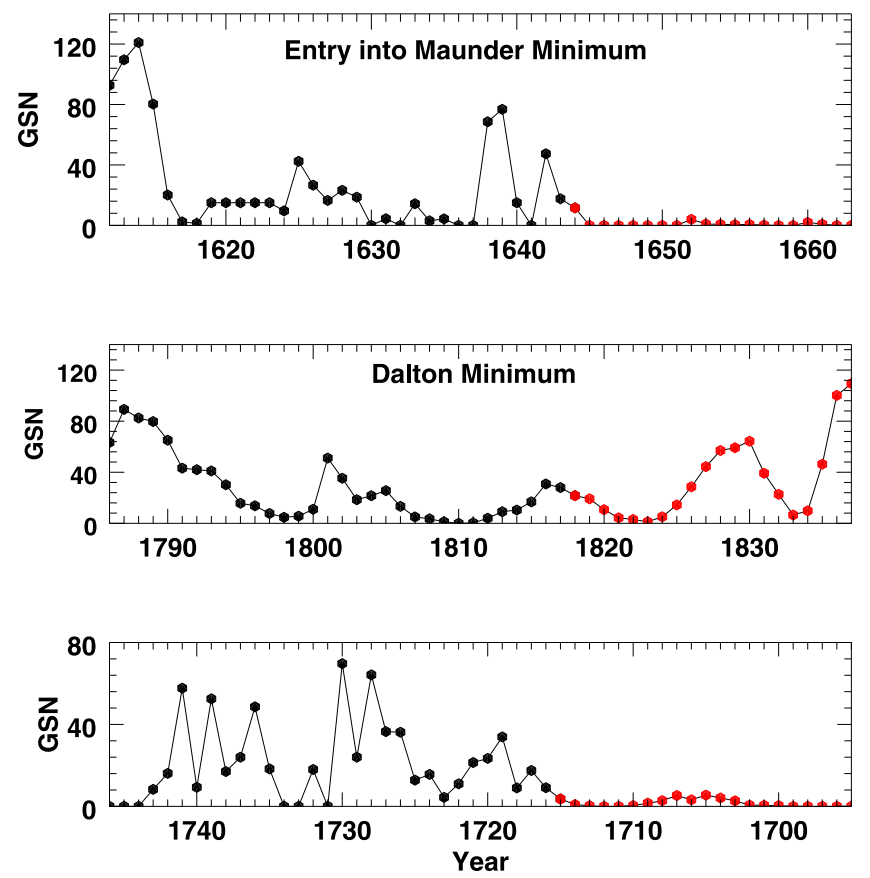

Figure 3. Trend of Group Sunspot Number (GSN) time series during the Sun's two significant magnetic grand minima episodes since $1600 \mathrm{CE}-$ Maunder Minimum and Dalton Minimum. The first and third panels show the GSN time series as the Sun enters and exits the Maunder Minimum, respectively. The second panel shows the GSN time series of the Sun during Dalton Minimum. The current trend of HD 4915 strongly resembles the trend of GSN as the Sun entered or exited the DM period, but if its low activity cycles continue for many more cycles or disappear entirely and become flat, it will be a relatively unambiguous Maunder Minimum analog.

on the mean activity of (presumably) non-MGM stars, and we do not know how the activity level during the transition to an MGM state relates to its mean out-of-MGM activity level.

Using the data we have, we would determine the age of the star to be between $4.3 \mathrm{Gyr}$ (at the global minimum, $S=0.176$ ) and $2.7 \mathrm{Gyr}$ (at the global maximum, $S=0.22$ ). We would calculate an age of 3.8 Gyrs using the mean of the data from just the last full cycle. This range of ages is consistent with the isochrone age, confirming that the star is likely to be younger than the Sun.

With $\log R_{\mathrm{HK}}^{\prime}=-4.877$ and $[\mathrm{M} / \mathrm{H}]=-0.2$, we note that it does not satisfy Saar \& Testa (2012)'s criteria of lying near the minimum $\log R_{\mathrm{HK}}^{\prime}-[\mathrm{M} / \mathrm{H}]$ trend-line yet. This could be explained if HD 4915 is younger than the Sun, which would cause its average activity levels to be higher or because it is still entering an MGM state, and thus shows depressed, but not yet MGM-level, activity. On the other hand, it is a bona fide dwarf as classified by its temperature and gravity, and its $\mathrm{HK}$ variation of $2.28 \%$ is close to the $2 \%$ limit set down by Saar \& Testa (2012).

\section{A Decreasing Cycle Strength Reminiscent of Solar MGM}

In this section, we analyze the S-Index time series of HD 4915 to categorize it as an MM candidate. As the top panel of Figure 2 shows, we see a decrease in the amplitude of the activity cycle over the three apparent maxima until the amplitude is almost negligible. By examining the first two cycles and assuming that the first measurements just capture the first cycle maximum, we estimate the period of the cycle to be four years and note the striking delay in the occurrence of the third maxima by almost two years. We also note that the second minimum appears to be significantly lower than the first minimum. These features of the S-Index time series strongly suggest a decreasing magnetic activity in the star.

To compare these features to that of the Sun, we look at the Sun's transitions into and out of MGM using its historical GSN record (reconstructed by Hoyt \& Schatten 1998). Figure 3 shows entry into MM (first panel), the Dalton Minimum (middle panel) and exit from the MM (last panel). The black symbols represent the section of the GSN trend that we are comparing with the past activity of HD 4915, and the red symbols represent the potential futures of the HD 4915 activity record if it were in an analogous state.

The transition of the Sun into MM is characterized by a steep and sudden drop in GSN across two normal cycles (Figure 3, top panel). GSNs then remains close to zero until toward the end of the $\mathrm{MM}$ at around $1700 \mathrm{CE}$, when it emerges from the MM over the course of two cycles and returns to its normal 11 year cycling state.

To date, the S-Index time series of HD 4915 most resembles the transition of the Sun into the Dalton Minimum in Figure 3 (middle panel, solid black dots). This transition is characterized by the decreasing amplitude of maxima over three cycles, with the third minimum occurring lower than the previous two (Figure 2).

Finally, we compare the HD 4915 S-Index time series to the exit of the Sun from the MM. This comparison is possible if we hypothesize that the entry and exit of the Sun's magnetic activity from a grand magnetic state are time symmetric. ${ }^{8}$ In the case of the MM event, the transition from MGM to a normal cycle as seen in Figure 3 (bottom panel) is characterized by the increasing amplitude of the magnetic activity (note the time reversed $x$-axis). This, too, bears some resemblance to the activity history of HD 4915 .

\section{Discussion and Conclusion}

The decline in the magnetic activity strength of HD 4915 is exciting because it renews the discussion of ways to identify MM candidates. As discussed in Section 1.2, there have been numerous attempts over the last few decades to set down the criteria for an MGM candidate using S-Indices; however, these criteria have been of uncertain utility because we lack directly comparable data of the Sun's chromospheric activity during its MGM episodes.

As indicated by Saar \& Testa (2012), the surest way to characterize a star in MGM would be by observing it enter a long period (at least longer than its typical cycle period) of flat activity or low cycling activity and transition out of this period into a normal activity cycle. Alternatively, we could also make such a characterization by observing a star transition into a normal cyclic state from an anomalously low state.

The discovery of a true MM analog would help us understand whether the state of the Sun's field during its MGM was a state of extraordinarily low global magnetic field strength, or just an extraordinarily weak and long series of magnetic maxima. Such an understanding would greatly inform searches for other MM analogs.

\footnotetext{
8 Such a symmetry is pure hypothesis on our part, as a way to generate a third example of an MGM-normal state transition from the Solar record.
} 
HD 4915 may, in fact, not be a star with a single, strong cycle period like the Sun. Indeed, we have inferred that it has a 4 years period under the assumption that our data capture the first partial cycle's maximum and what appears to be around two full "cycles" of varying morphology. As has been shown by Metcalfe et al. (2016), many stars with cycle periods $<5$ years show stochastic or multi-period behavior that might be a better analogy to HD 4915 (see, for instance, HD 76151 in Figures 4.5 and B.10 of Egeland 2017, as well as HD 190406 in Figure 7, left column, second from top; Hall et al. 2007). This underscores the need for future observations of this star to determine its magnetic behavior.

We recommend immediate and future observations of chromospheric and coronal cooling indicators (such as the $\mathrm{H}$ $\& \mathrm{~K}$ emission lines and $\mathrm{X}$ rays) for at least the next decade (two cycles of the star), especially as there is strong evidence that HD 4915 is entering a state of MGM right now. The Transiting Exoplanet Survey Satellite (TESS) or other future space-based photometric missions might be able to determine if the star indeed has a spot-free photosphere.

We thank the referee for a constructive report that improved this Letter, and for pointing us to the Donahue et al. identification of HD 3651 as an MGM candidate.

We thank Eric Mamajek for useful discussions and for hunting down the early use of the term "Dalton Minimum.", We also thank Thomas Beatty, Fabienne Bastien, Mark Giampapa, David Soderblom, and Larry Ramsey for useful discussions that improved the Letter.

The Center for Exoplanets and Habitable Worlds is supported by the Pennsylvania State University, the Eberly College of Science, and the Pennsylvania Space Grant Consortium.

The data presented herein were obtained at the W.M. Keck Observatory, which is operated as a scientific partnership among the California Institute of Technology, the University of California and the National Aeronautics and Space Administration. The Observatory was made possible by the generous financial support of the W.M. Keck Foundation.

This research has made use of NASA's Astrophysics Data System Bibliographic Services, the SIMBAD database, operated at CDS, Strasbourg, France, and the AstroBetter blog and wiki.

This material is based upon work supported by the NSF Postdoctoral Research Fellowship in Biology under grant No. AST-1602662.

Facility: Keck:I (HIRES).

\section{ORCID iDs}

Shivani P. Shah (1) https://orcid.org/0000-0002-3367-2394

Jason T. Wright (1) https://orcid.org/0000-0001-6160-5888

Howard Isaacson (ib https://orcid.org/0000-0002-0531-1073

Andrew W. Howard (i) https://orcid.org/0000-0001-

8638-0320

Jason L. Curtis (i) https://orcid.org/0000-0002-2792-134X

\section{References}

Baliunas, S., \& Jastrow, R. 1990, Natur, 348, 520

Baliunas, S. L., Donahue, R. A., Soon, W. H., et al. 1995, ApJ, 438, 269

Brewer, J. M., Fischer, D. A., Valenti, J. A., \& Piskunov, N. 2016, ApJS, 225, 32

Butler, R. P., Vogt, S. S., Laughlin, G., et al. 2017, AJ, 153, 208

Charbonneau, P. 2014, ARA\&A, 52, 251

Cullers, K. 2000, in ASP Conf. Ser. 213, Bioastronomy, ed. G. Lemarchand \& K. Meech (San Francisco, CA: ASP), 99

Curtis, J. L. 2017, AJ, 153, 275

do Nascimento, J. D., Jr., Canto Martins, B. L., Melo, C. H. F., Porto de Mello, G., \& De Medeiros, J. R. 2003, A\&A, 405, 723

Donahue, R. A., Baliunas, S. L., Soon, W. H., \& McMillan, F. M. 1995, in IAU Symp. 176 (Cambridge: Cambridge Univ. Press), 72

Eddy, J. A. 1976, Sci, 192, 1189

Eddy, J. A. 1982, in Proc. Southwest Regional Conf. for Astronomy and Astrophysics

Egeland, R. 2017, PhD thesis, Montana State Univ. Bozeman

Giampapa, M. S., Hall, J. C., Radick, R. R., \& Baliunas, S. L. 2006, ApJ, 651,444

Hall, J. C., Lockwood, G. W., \& Skiff, B. A. 2007, AJ, 133, 862

Henry, T. J., Soderblom, D. R., Donahue, R. A., \& Baliunas, S. L. 1996, AJ, 111, doi:10.1086/117796

Howard, A. W., Johnson, J. A., Marcy, G. W., et al. 2010, ApJ, 721, 1467

Hoyt, D. V., \& Schatten, K. H. 1998, SoPh, 181, 491

Isaacson, H., \& Fischer, D. 2010, ApJ, 725, 875

Kocharov, G. E. 1986, in ESA Special Publication 251, Plasma Astrophysics, ed. T. D. Guyenne \& L. M. Zeleny

Leighton, R. B. 1959, ApJ, 130, 366

Mamajek, E. E., \& Hillenbrand, L. A. 2008, ApJ, 687, 1264

Metcalfe, T. S., Egeland, R., \& van Saders, J. 2016, ApJL, 826, L2

Noyes, R. W., Hartmann, L. W., Baliunas, S. L., Duncan, D. K., \& Vaughan, A. H. 1984, ApJ, 279, 763

Pavlenko, Y. V., Jenkins, J. S., Jones, H. R. A., Ivanyuk, O., \& Pinfield, D. J. 2012, MNRAS, 422, 542

Perryman, M. A. C., Lindegren, L., Kovalevsky, J., et al. 1997, A\&A, 323, L49 Prša, A., Harmanec, P., Torres, G., et al. 2016, AJ, 152, 41

Saar, S. H., \& Testa, P. 2012, in IAU Symp. 286, Comparative Magnetic Minima: Characterizing Quiet Times in the Sun and Stars, ed. C. H. Mandrini \& D. F. Webb (Cambridge: Cambridge Univ. Press), 335

Siscoe, G. L. 1980, RvGSP, 18, 647

Skumanich, A., Smythe, C., \& Frazier, E. N. 1975, ApJ, 200, 747

Soubiran, C., Le Campion, J.-F., Brouillet, N., \& Chemin, L. 2016, A\&A, 591, A118

Vaughan, A. H., Preston, G. W., \& Wilson, O. C. 1978, PASP, 90, 267

Vogt, S. S., Allen, S. L., Bigelow, B. C., et al. 1994, Proc. SPIE, 2198, 362

Wilson, O. C. 1968, ApJ, 153, 221

Wright, J. T. 2004, AJ, 128, 1273

9 https://twitter.com/ericmamajek/status/929550539834007552 